\title{
Estrutura e organização espacial de duas comunidades de anuros do bioma Pampa
}

\author{
Victor M. Lipinski ${ }^{1} \&$ Tiago G. dos Santos ${ }^{2}$
}

\begin{abstract}
1. Programa de Pós-Graduação em Biodiversidade Animal, Universidade Federal de Santa Maria, Laboratório de Herpetologia, Departamento de Biologia, Av. Roraima s/n, Prédio 17, sala 1140, Camobi, 97105-900 Santa Maria, RS, Brasil. (lipinskivictor@hotmail.com)

2. Universidade Federal do Pampa, Av. Antônio Trilha, 1847, Centro, 97300-000 São Gabriel, RS, Brasil.
\end{abstract}

\begin{abstract}
Structure and spatial organization of two anuran communities of the Pampa biome. The Pampa is the smallest Brazilian biome and is one of the most endangered, due to the expansion of the agropastoril cultures and the silviculture; is one of the Brazilian biomes with the lowest representation on the conservation unities system. In this study are presented information about the structure and spatial organization of anuran communities of two physiographic regions, from the four in which the biome is divided. Were sampled ten waterbodies in each region between the months of September 2011 and August 2012. Were registered 24 species characterized as generalist, with wide distribution and typical of the open areas. The species accumulation curve showed an assintota after the $12^{\circ}$ month of sampling. The similarity analysis showed a segregation in the structure of both communities that were influenced by the environmental landscape descriptors.
\end{abstract}

KEYWORDS. Amphibia, anurofauna, ponds, community ecology.

RESUMO. O Pampa é o menor bioma brasileiro e está entre os mais ameaçados devido ao rápido avanço das culturas agropastoris e da silvicultura; é um dos biomas com menor representação no sistema de unidades de conservação. Neste estudo são apresentadas informações sobre a estrutura e a organização espacial das comunidades de anuros de duas das quatro regiões fisiográficas em que o bioma é dividido. Foram amostrados dez corpos d'água em cada região entre os meses de setembro de 2011 e agosto de 2012. Foram registradas 24 espécies caracterizadas como generalistas, de ampla distribuição e típicas de ambientes abertos. A curva de acumulação de espécies apresentou a formação de uma assíntota após o $12^{\circ}$ mês de amostragem. A análise de similaridade mostrou uma segregação na estrutura das duas comunidades, que foram influenciadas pelos descritores ambientais e da paisagem.

PALAVRAS-CHAVE. Amphibia, anurofauna, poças, ecologia de comunidades.

O Pampa é o menor bioma brasileiro e está representado apenas no estado do Rio Grande do Sul, onde ocupa aproximadamente $62 \%$ do território; fora do Brasil, ocorre em parte da Argentina e todo o território do Uruguai (BoLDRINI et al., 2010). No entanto, o nível de desenvolvimento econômico e social desta região do estado historicamente tem levado o bioma a um processo constante de conversão de sua área original por paisagens agrícolas, financiadas por incentivos fiscais por parte do Governo do Estado (P. Binkowski, dados inéditos). Atualmente a fisionomia campestre original já foi descaracterizada em torno de $50 \%$ devido a ações humanas de urbanização e demais atividades econômicas (HASENACK et al., 2007). Segundo BoldRINI et al. (2010). Os tipos de vegetação, solos e a própria riqueza de condições geológicas e geomorfológicas, aspectos hidrológicos e a ordem climática tornam o bioma Pampa um território heterogêneo, onde as áreas menos degradadas estão distribuídas em mosaicos, nos locais de topografia acentuada e com solos pouco férteis (OverBeCK et al., 2007; BeHLING et al., 2009; BENCKE, 2009; Cordeiro \& Hasenack, 2009; Guadagnin et al., 2009; Gautreau \& Vélez, 2011). Assim, o conhecimento acerca da biodiversidade dos campos nativos ainda é incipiente, tornando baixo o número de áreas efetivamente protegidas em Unidades de Conservação (Overbeck et al., 2007).

Os estudos envolvendo ecologia de comunidades de anuros existentes no Bioma Pampa, historicamente, estão concentrados na região da Planície Costeira (e.g., Colombo et al., 2008; MaltchiK et al., 2008; Moreira et al., 2008; Machado \& Maltchiк, 2009; Machado et al., 2012; OLIVEIRA et al., 2013), enquanto as fisionomias campestres interioranas permaneceram pouco exploradas exceto pelos estudos de Santos et al. (2008) na Depressão Central, Bотн et al. (2011) na Serra do Sudeste e Di-Bernardo et al. (2002) na região da Campanha. Apesar do crescente número de estudos, o conhecimento acerca dos padrões de composição, diversidade e distribuição espacial dos anuros desta região ainda são escassos. Atualmente, existem aproximadamente 50 espécies de anfíbios conhecidas para o bioma Pampa (GARCIA et al., 2007) e, dentre estas, aproximadamente oito são consideradas endêmicas (BENCKE et al., 2009).

Existem diversos fatores históricos (VALDujo et al., 2011), espaciais (IUCN, 2012), pressões predativas (tanto por vertebrados quanto por invertebrados) e de competição que modificam padrões de composição e endemismo de espécies e assim, contribuindo para a distribuição diferencial destas nos locais de ocorrência. Para os anfíbios do Pampa, a heterogeneidade da paisagem campestre (BolDRINI et $a l ., 2010)$ proporciona um mosaico de corpos d'água e amplia o número de recursos a serem utilizados pelas espécies (Nogueira et al., 2009), facilitando a dispersão entre os corpos d'água e proporcionando um fator ambiental importante na determinação dos padrões de riqueza local 
(Hawkins et al., 2007). Desta forma, a manutenção das áreas abertas e dos mosaicos formados pela vegetação, relevo e pelos corpos d'água são fundamentais para a manutenção dos nichos responsáveis pelas espécies nos locais. Diante deste cenário, objetiva-se ampliar o conhecimento dos padrões de distribuição espacial, riqueza e composição da anurofauna do bioma Pampa em duas áreas com diferentes tipos de exploração da terra.

\section{MATERIAL E MÉTODOS}

Área de estudo. O estudo foi realizado em duas das quatro regiões fisiográficas do bioma Pampa (e.g. Planície Costeira, Planalto da Campanha, Depressão Central, e Serra do Sudeste), Depressão Central (DC) e Serra do Sudeste (SS), localizadas nos municípios de

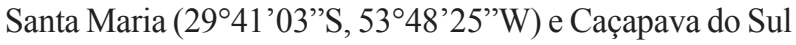
(30³0'44'S, 5329'29'W), respectivamente. A Depressão Central apresenta relevo suavemente ondulado, não ultrapassando os $400 \mathrm{~m}$ de altitude. A composição rochosa é representada por formações Paleozoicas do Carbonífero Superior. Segundo HASENACK et al. (2010), a vegetação é categorizada como Campos com Andropogôneas e Compostas; o clima, segundo a classificação de MALuF (1999), é do tipo subtropical úmido (ST UM) com temperaturas médias mínimas chegando aos $13,8^{\circ} \mathrm{C}$ e média anual de chuvas de $1708 \mathrm{~mm}$ (KRAPOVICKAS \& Di Giacomo, 1998; Porto, 2002; Overbeck et al., 2007). A região da Serra do Sudeste apresenta um relevo fortemente ondulado, com média de altitudes variando entre 400 e 600 m (RAMBo, 1956; PoRTO, 2002). A vegetação é composta por campos e matas de galeria, parque e capões (VIEIRA \&
RANGel, 1984), com gramíneas rasteiras e solos expostos especialmente nas encostas dos morros, nestes locais mais secos, é comum a predominância de espécies como a barbade-bode (BoldrinI, 1997). O clima é do tipo temperado úmido (TE UM) com temperaturas médias mínimas de $11,4^{\circ} \mathrm{C}$ e médias anuais de chuva de $1588 \mathrm{~mm}$ (Fig. 1).

Hábitats amostrados. Os corpos d'água foram selecionados de acordo com a distância entre os mesmos e as características da paisagem no qual estão inseridos. Desta forma, dez corpos d'água foram selecionados na Serra do Sudeste (distantes em média 4,45 $\pm 2,30 \mathrm{~km}$ ) e 11 na Depressão Central (distantes em média 4,65 $\pm 2,41$ $\mathrm{km})$ (Tab. I). Estes corpos d'água foram caracterizados por descritores ambientais da seguinte forma: 1) Área total da poça; 2) Altitude; 3) Hidroperíodo; 4) Movimentação da água; 5) Perfis de inclinação da margem; 6) Número de tipos de margem; 7) Cobertura vegetal no espelho d'água; 8) Número de perfis de vegetação emergente; 9) Tipo de matriz; 10) Profundidade e 11) Origem da poça. Adicionalmente, cinco parâmetros físico-químicos da água foram medidos (três medidas para cada parâmetro): A) Temperatura; B) pH; C) Oxigênio dissolvido; D) Condutividade elétrica e E) Turbidez; para tais medições foi utilizada uma sonda multiparâmetros (SANTos et al., 2007; VAsConcelos et al., 2009).

Amostragem. As áreas foram estudadas mensalmente de setembro de 2011 a agosto de 2012, totalizando 12 amostragens de aproximadamente 24 dias. Para fins de padronização do esforço amostral e evitar os possíveis ruídos das variações diárias dos parâmetros da água, ao início das amostragens em cada campanha a ordem de amostragem das poças era escolhida aleatoriamente

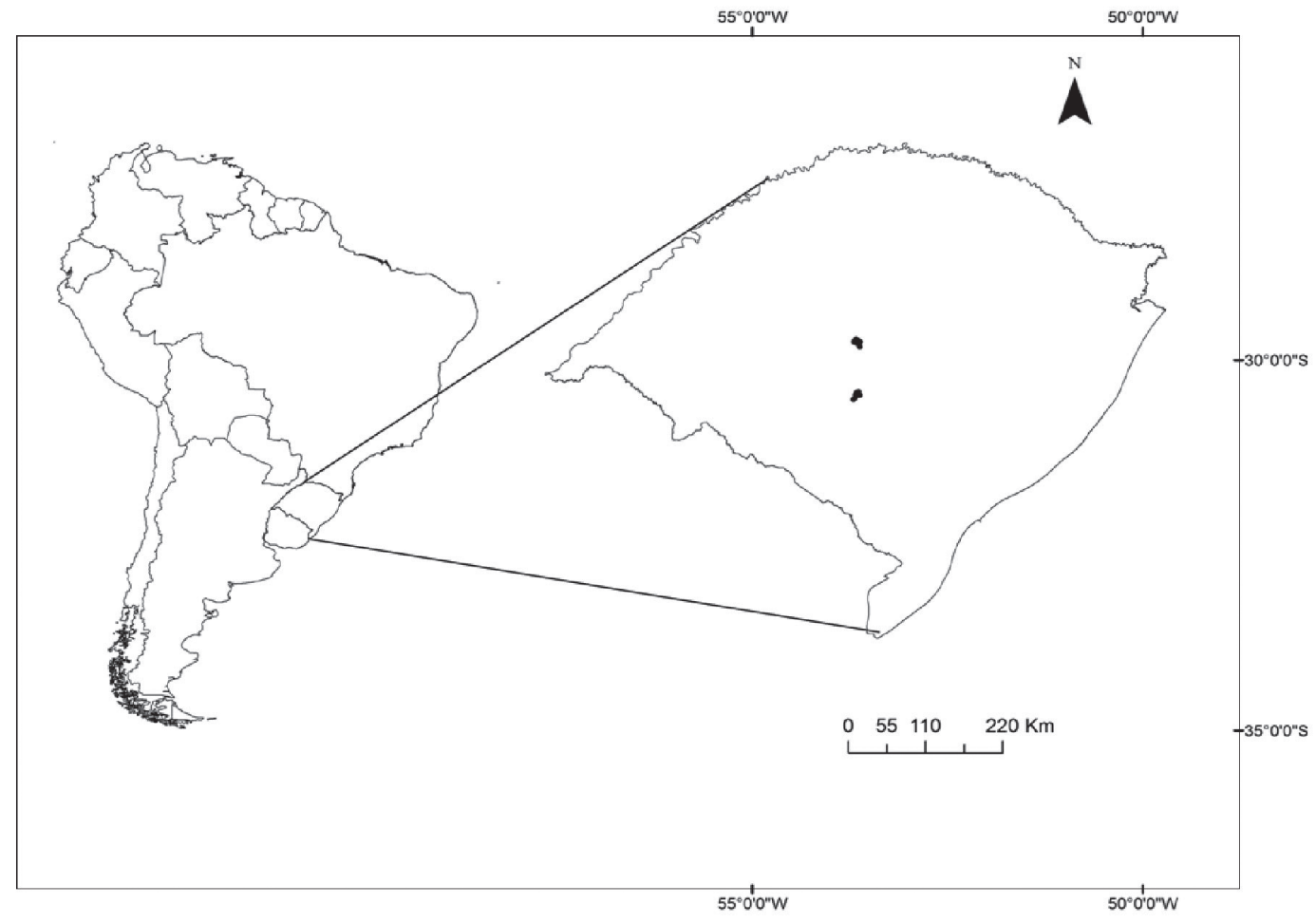

Fig. 1. Localização das áreas de estudo, municípios de Caçapava do Sul e Santa Maria, Rio Grande do Sul, Brasil. 
Tab. I. Sítios de amostragem de anfíbios anuros nos municípios de Santa Maria e Caçapava do Sul, Rio Grande do Sul, Brasil as respectivas coordenadas geográficas.

\begin{tabular}{cccc}
\hline Poças & Localização & Latitude & Longitude \\
\hline DC1 & Santa Maria & $-29,824231$ & $-53,621175$ \\
DC2 & Santa Maria & $-29,76135$ & $-53,612595$ \\
DC3 & Santa Maria & $-29,749954$ & $-53,640313$ \\
DC4 & Santa Maria & $-29,720978$ & $-53,665122$ \\
DC5 & Santa Maria & $-29,740775$ & $-53,659486$ \\
DC6 & Santa Maria & $-29,747425$ & $-53,693539$ \\
DC7 & Santa Maria & $-29,744836$ & $-53,641351$ \\
DC8 & Santa Maria & $-29,764281$ & $-53,692792$ \\
DC9 & Santa Maria & $-29,774425$ & $-53,661914$ \\
DC10 & Santa Maria & $-29,761294$ & $-53,657658$ \\
DC11 & Santa Maria & $-29,823407$ & $-53,622085$ \\
SS1 & Caçapava do Sul & $-30,447439$ & $-53,658928$ \\
SS2 & Caçapava do Sul & $-30,484508$ & $-53,653214$ \\
SS3 & Caçapava do Sul & $-30,480451$ & $-53,619816$ \\
SS4 & Caçapava do Sul & $-30,431036$ & $-53,634999$ \\
SS5 & Caçapava do Sul & $-30,436131$ & $-53,655898$ \\
SS6 & Caçapava do Sul & $-30,452549$ & $-53,663332$ \\
SS7 & Caçapava do Sul & $-30,474318$ & $-53,678074$ \\
SS8 & Caçapava do Sul & $-30,508608$ & $-53,682252$ \\
SS9 & Caçapava do Sul & $-30,5161$ & $-53,682056$ \\
SS10 & Caçapava do Sul & $-30,534741$ & $-53,700234$ \\
\hline
\end{tabular}

tanto por local quanto por horário e o perímetro destas percorrido por duas pessoas utilizando métodos de busca auditiva e visual (ver busca em sítios de reprodução, ScOTT JR. \& WoOdWARd, 1994) (GotTSBerger \& Gruber, 2004; VASCONCElos \& RossA-FERES, 2005; SANTOS et al., 2007). Indivíduos localizados vocalizando foram contabilizados para riqueza e dados de abundância; os indivíduos avistados perto das poças ou que não estivessem vocalizando foram considerados apenas para estimativas de riqueza das áreas. Adicionalmente, foram coletados anuros em estágios larvais (para dados de riqueza das áreas) e potenciais predadores de larvas (vertebrados e invertebrados aquáticos, identificados em nível de família) com o auxílio de um puçá de malha de arame de $3 \mathrm{~mm}$ e cabo longo. Para isto, o puçá foi utilizado ao longo do perímetro das poças (SANTOS et al., 2007). Espécimes-testemunho foram coletados e eutanasiados com o uso de Lidocaína a $10 \%$ e tombados na Coleção de Herpetologia da Universidade Federal de Santa Maria (ZUFSM, ICMBIO Licença n 29508/3).

Análises de dados. Para determinar a suficiência amostral nas duas áreas estudadas, foi gerada uma curva de acumulação de espécies baseada na riqueza (adultos e larvas) (100 randomizações, Gotelli \& Colwell, 2001). Para acessar dados de uso do habitat e a relação dos descritores ambientais com a estrutura da comunidade de anuros (medida através de abundância dos indivíduos adultos em atividade de vocalização), foi utilizada uma análise "Bioenv", que determina a melhor combinação de variáveis que explicam as correlações não paramétricas entre os arranjos de descritores ambientais e a matriz que representa a estrutura da comunidade (CLARKE \& Ainsworth, 1993). Um teste de Mantel foi aplicado para detectar influência da distância geográfica sobre a estrutura das comunidades (matriz de abundância) e uma análise de espécies indicadoras (abundância e riqueza) (ISA, DUFRÊNE \& LEGENDRE, 1997) foi utilizada para detectar a preferência das espécies quanto às regiões amostradas com base em dados de frequência de ocorrência e abundância ao longo de dois grupos: (i) amostras da Depressão Central e (ii) amostras da Serra do Sudeste. A similaridade foi testada através de uma ANOSIM (CLARK \& WARWICK, 2001a,b) aplicando o coeficiente de similaridade de Bray-Curtis, considerando-se a máxima abundância de machos em atividade de vocalização em cada poça e uma análise de Escalonamento Multidimensional Não Métrico (NMDS) para ilustrar de forma gráfica os padrões de distribuição. O valor de stress foi utilizado como medida de representatividade da matriz pelo método de ordenação (Warwick \& Clarke, 2001b). Por fim, uma análise de regressão foi utilizada para verificar se existe relação da presença de predadores com a abundância e riqueza de anuros e uma matriz de correlação de variáveis foi construída a fim de detectar a existência de colinearidade nas variáveis ambientais para que estas fossem excluídas a priori das análises. Todas as análises foram conduzidas no ambiente estatístico $\mathrm{R}$ utilizando o pacote vegan ( $\mathrm{R}$ Core TEam, 2012).

\section{RESULTADOS}

Riqueza e abundância de espécies de anuros. Ao longo das duas áreas, foram registradas 24 espécies distribuídas em 12 gêneros e cinco famílias: Bufonidae (2), Odontophrynidae (1), Hylidae (10), Leptodactyliade (10) e Microhylidae (1). Destas espécies, 15 foram também registradas em estágio larval. Na região da Serra do Sudeste, Scinax granulatus, Physalaemus gracilis e Pseudis minuta foram responsáveis pelas maiores abundâncias com relação aos indivíduos adultos e, em estágio larval, Hypsiboas pulchellus, S. granulatus, Odontophrynus americanus foram as mais abundantes. Na Depressão Central, adultos de Dendropsophus minutus, D. sanborni e $S$. squalirostris 
e os estágios larvais de $S$. fuscovarius, $S$. granulatus e $S$. squalirostris foram mais abundantes, respectivamente (Tab. II). Com o registro destas 24 espécies, ao final das 12 amostragens a curva de acumulação de espécies (Fig. 2) mostrou uma tendência à assíntota para ambas regiões, descartando assim um incremento significativo no número de espécies.

Similaridade das Comunidades. Com relação à similaridade das comunidades de anuros das duas regiões fisiográficas estudadas, as análises de similaridade e a ordenação demostraram a existência de uma diferença significativa na composição de espécies $\left(\mathrm{R}^{2}=0.65, p=\right.$ 0.001 ), responsável pela formação de dois grupos distintos $($ NMDS, stress $=0,11)$ (Fig. 3).

Preferência de hábitat das espécies. A análise de espécies indicadoras demonstrou que espécies como Physalaemus biligonigerus (Cope, 1861) e Scinax squalirostris (A. Lutz, 1925) (Indval $=27 \%$ e $82 \%$ respectivamente) estão mais relacionadas aos ambientes da Depressão Central e espécies como P. gracilis (Boulenger, 1883), Rhinella schneideri (Werner, 1984) e S. uruguayus (Schmidt, 1944) $($ Indval $=90 \%, 48 \%$ e 40\% respectivamente) estão mais relacionadas à Serra do Sudeste.
Influências bióticas e abióticas. A riqueza de famílias de predadores aquáticos de larvas de anuros não mostrou nenhuma relação com incremento ou decréscimo na abundância ou riqueza destes. A estrutura destas comunidades foi significativamente afetada pelos parâmetros físicos e químicos da água, bem como pelos descritores ambientais mensurados nos locais. $\mathrm{Na}$ comunidade amostrada na Depressão Central a temperatura da água $\left({ }^{\circ} \mathrm{C}\right), \mathrm{o} \mathrm{pH}$, o tipo de matriz campestre em que a poça está inserida e número de perfis de vegetação emergente no espelho d'água foram o conjunto de variáveis responsável pelo maior coeficiente de correlação com a matriz biótica (r $=0,7, p=<0,005)$. Na estrutura da comunidade da Serra do Sudeste, o maior coeficiente de correlação foi apresentado pelo conjunto de variáveis taxa de oxigênio dissolvido na água $(\mathrm{mg} / \mathrm{L})$, condutividade elétrica $(\mathrm{msc} / \mathrm{s})$, variação de hidroperíodo, cobertura vegetal no espelho d'água e número de perfis de vegetação emergente no espelho d'água ( $\mathrm{r}$ $=0.38, \mathrm{p}=<0,005$ ). Por fim, o teste de Mantel mostrou que não existe influência da distância geográfica entre as poças amostradas, tanto na Serra do Sudeste quanto na Depressão Central $(r=-0,04 ; p=0,59$ e $r=0,11 ; p=0,27$ respectivamente).

Tab. II. Lista das espécies de anuros registrados nos municípios de Caçapava do Sul (SS) e Santa Maria (DC), Rio Grande do Sul, Brasil, nos meses de setembro de 2011 a agosto de 2012 (*, indivíduo somente avistado).

\begin{tabular}{|c|c|c|c|c|c|c|}
\hline & \multicolumn{2}{|c|}{ Região Fisiográfica } & \multicolumn{2}{|c|}{ Adulto } & \multicolumn{2}{|c|}{ Larva } \\
\hline & SS & $\mathrm{DC}$ & SS & $\mathrm{DC}$ & $\mathrm{SS}$ & $\mathrm{DC}$ \\
\hline \multicolumn{7}{|l|}{ BUFONIDAE } \\
\hline Rhinella achavali (Maneyro, Arrieta \& de Sá, 2004) & $\mathrm{X}$ & & & & & \\
\hline Rhinella schneideri (Werner, 1984) & $\mathrm{X}$ & & $\mathrm{X}$ & & & \\
\hline \multicolumn{7}{|l|}{ CYCLORAMPHIDAE } \\
\hline Odontophrynus americanos (Duméril \& Bibron, 1841) & $\mathrm{X}$ & $\mathrm{X}$ & $\mathrm{X}$ & $\mathrm{X}$ & $\mathrm{X}$ & $\mathrm{X}$ \\
\hline \multicolumn{7}{|l|}{ HYLIDAE } \\
\hline Dendropsophus minutus (Peters, 1872) & $\mathrm{X}$ & $\mathrm{X}$ & $\mathrm{X}$ & $\mathrm{X}$ & $\mathrm{X}$ & $\mathrm{X}$ \\
\hline Dendropsophus sanborni (Schmidt, 1944) & $\mathrm{X}$ & $\mathrm{X}$ & $\mathrm{X}$ & $\mathrm{X}$ & $\mathrm{X}$ & $\mathrm{X}$ \\
\hline Hypsiboas pulchellus (Duméril \& Bibron, 1841) & $\mathrm{X}$ & $\mathrm{X}$ & $\mathrm{X}$ & $\mathrm{X}$ & $\mathrm{X}$ & $\mathrm{X}$ \\
\hline Phylomedusa iheringii Boulenger, 1885 & $\mathrm{X}$ & $X$ & $\mathrm{X}$ & $X$ & $\mathrm{X}$ & \\
\hline Pseudis minuta Günter, 1859 & $\mathrm{X}$ & $X$ & $\mathrm{X}$ & $X$ & $\mathrm{X}$ & $\mathrm{X}$ \\
\hline Scinax berthae (Barrio, 1962) & & $X$ & & $X$ & & $\mathrm{X}$ \\
\hline Scinax fuscovarius (A. Lutz, 1925) & $\mathrm{X}$ & $X$ & $\mathrm{X}$ & $X$ & $\mathrm{X}$ & $\mathrm{X}$ \\
\hline Scinax granulatus (Peters, 1871) & $\mathrm{X}$ & $X$ & $\mathrm{X}$ & $X$ & $\mathrm{X}$ & $\mathrm{X}$ \\
\hline Scinax squalirostris (A. Lutz, 1925) & & $X$ & & $X$ & $\mathrm{X}$ & $\mathrm{X}$ \\
\hline Scinax uruguayus (Schmidt, 1944) & $\mathrm{X}$ & & $\mathrm{X}$ & & $\mathrm{X}$ & \\
\hline \multicolumn{7}{|l|}{ LEPTODACTYLIDAE } \\
\hline \multicolumn{7}{|l|}{ Leptodactylus chaquensis (Cei, 1950) * } \\
\hline Leptodactylus fuscus (Schneider, 1799) & & $\mathrm{X}$ & & $\mathrm{X}$ & & $\mathrm{X}$ \\
\hline Leptodactylus gracilis (Duméril \& Bibron, 1841) & & & $\mathrm{X}$ & & & \\
\hline Leptodactylus latinasus (Jiménez de la Espada, 1875) & $X$ & $\mathrm{X}$ & $\mathrm{X}$ & $\mathrm{X}$ & & \\
\hline Leptodactylus latrans (Linaeus, 1758) & $\mathrm{X}$ & $\mathrm{X}$ & $\mathrm{X}$ & $X$ & $\mathrm{X}$ & \\
\hline Leptodactylus mystacinus (Burmeister, 1861) & $\mathrm{X}$ & $\mathrm{X}$ & $\mathrm{X}$ & $X$ & & \\
\hline Physalaemus biligonigerus (Cope, 1961) & $\mathrm{X}$ & $X$ & & $X$ & & $\mathrm{X}$ \\
\hline Physalaemus cuvieri Fitzinger, 1826 & $\mathrm{X}$ & $X$ & $\mathrm{X}$ & $X$ & $\mathrm{X}$ & $\mathrm{X}$ \\
\hline Physalaemus gracilis (Boulenger, 1883) & & $X$ & $\mathrm{X}$ & & $\mathrm{X}$ & \\
\hline Pseudopaludicola falcipes (Cope, 1862) & $X$ & $\mathrm{X}$ & $\mathrm{X}$ & $X$ & $\mathrm{X}$ & $\mathrm{X}$ \\
\hline \multicolumn{7}{|l|}{ MICROHYLIDAE } \\
\hline Elachistocleis bicolor (Guerin-Méneville, 1838) & $\mathrm{X}$ & $\mathrm{X}$ & $\mathrm{X}$ & $\mathrm{X}$ & $\mathrm{X}$ & $\mathrm{X}$ \\
\hline
\end{tabular}




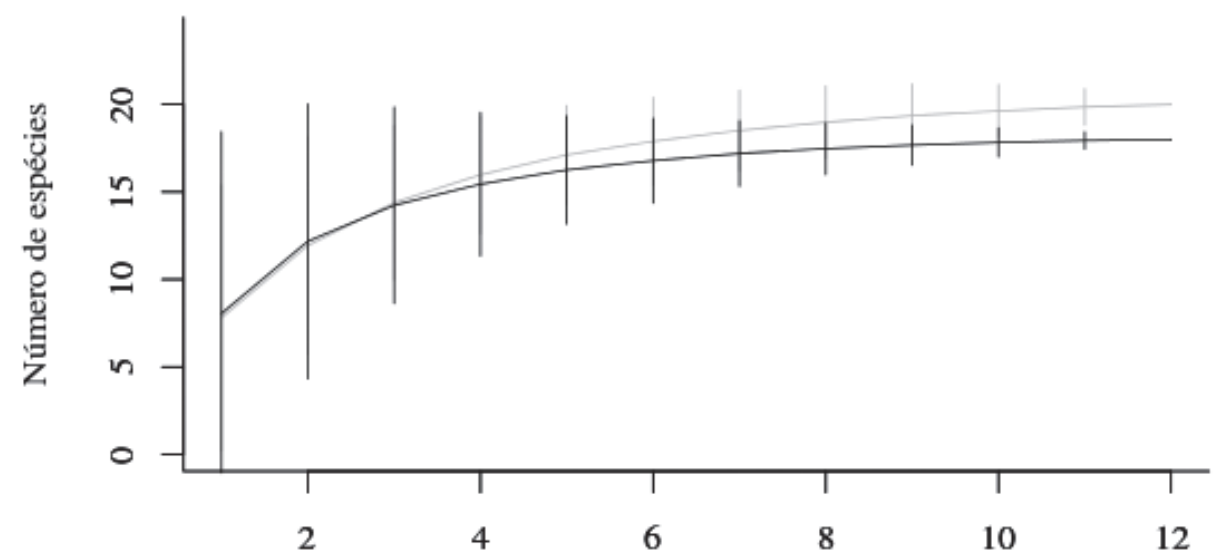

Número de amostras

Fig. 2. Curvas de acumulação de espécies de anfíbios nas áreas amostradas, municípios de Santa Maria (linha cinza) e Caçapava do Sul (linha preta), Rio Grande do Sul, Brasil.

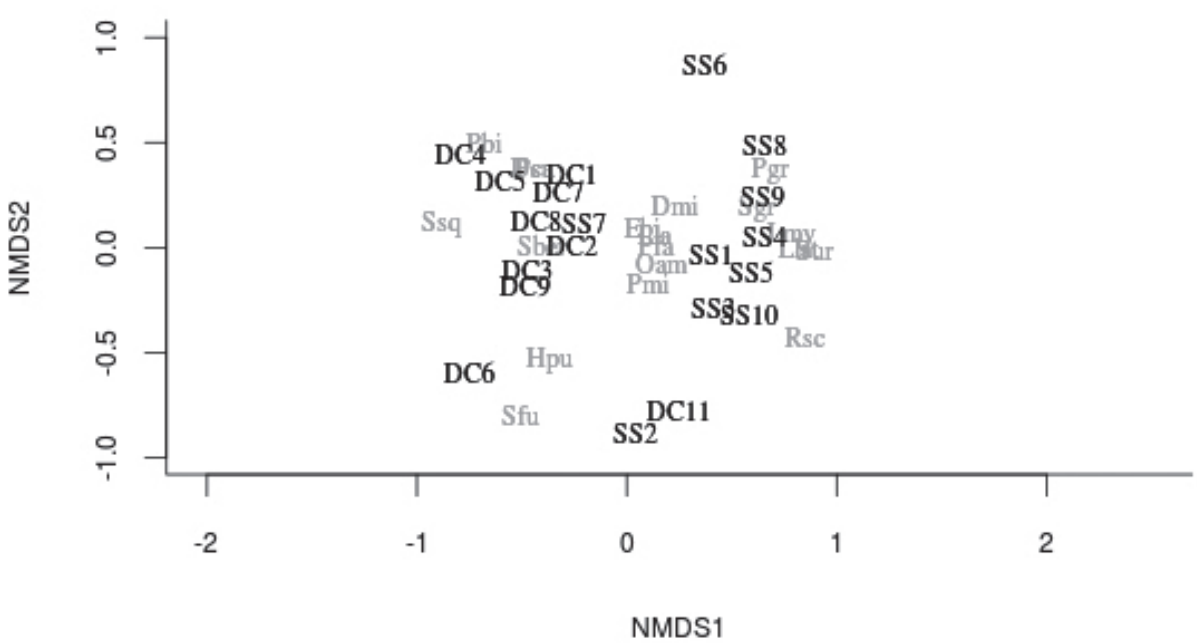

Fig. 3. Gráfico de ordenação por NMDS com a similaridade da composição das comunidades de anuros nos municípios de Santa Maria (DC 1-11) e Caçapava do Sul (SS 1-10), Rio Grande do Sul, Brasil (stress=0,11).

\section{DISCUSSÃO}

Foram registradas 24 espécies de anuros para as duas áreas estudadas, sendo um número esperado dado que outros estudos na região dos campos sulinos como SAntos et al. (2008), Bотн et al. (2009), TRINDADE et al. (2010), registraram riqueza semelhante de espécies, aproximadamente $25 \%$ de riqueza conhecida para o estado do Rio Grande do Sul (Quintela et al., 2009; Iop et al., 2011). No entanto, das 24 espécies registradas apenas duas são endêmicas do bioma Pampa (Phyllomedusa iheringii e Physalaemus gracilis) (Kwet \& Di-Bernardo, 1998; Maneyro \& Langone, 2001; Santos et al., 2008; Вотн et al., 2011; Moreira \& MaLtichiK, 2012). Pelo fato de que muitas das espécies encontradas nestes estudos têm a reprodução concentrada em curtos períodos (SANTOS et $a l .$, 2007) (reprodução explosiva), é esperado que o padrão de ocorrência de algumas tenha sido subamostrado (e.g. gênero Melanophryniscus).
Nos últimos anos, o número de estudos visando entender as interações entre os parâmetros abióticos e as comunidades de anuros tem crescido (e.g. Вотн et al., 2009; Oda et al., 2009; Iop et al., 2011; Gambale et al., 2014) e alguns descritores ambientais estudados (e.g. hidroperíodo, condutividade elétrica, $\mathrm{pH}$, presença de predadores e cobertura vegetal) vem revelando um padrão recorrente de influência direta e/ou indireta na estrutura das comunidades (BASTAZINI et al., 2007; VASCONCELOS et al., 2010; XaVier \& NAPOLI, 2011; Moreira \& MALtchiK, 2012), logo, a diferença já reconhecida (geologia, uso da terra, altitude, clima, dentre outros) entre as duas regiões amostradas pode, de alguma forma refletir uma estrutura diferente de anurofauna em cada uma delas.

Ainda que a maior parte das espécies registradas tenha ampla distribuição (CAMARGO et al., 2005), o fato de terem sido registrados números semelhantes de espécies terrícolas, arborícolas e aquáticas, indica que o ambiente não é inteiramente homogêneo, por mais que as regiões 
estudadas estejam situadas em matrizes de campo, o que em teoria confere às paisagens uma característica homogênea (Vasconcelos \& Rossa-Feres, 2008; Vasconcelos et al., 2009; SiLVA et al., 2011). As regiões Depressão Central e Serra do Sudeste são caracterizadas por diferentes tipos de ambientes campestres, o que proporciona aos anuros maior disponibilidade de microambientes a serem explorados, permitindo uma riqueza elevada (para a região dos campos).

Este tipo de variação (de fauna) entre dois locais, ligado à estrutura da paisagem, pode ser detectado por resultados semelhantes ao do presente estudo, quando uma das principais pressões bióticas (predação ver HERo et al., 1998) não mostra relações com a abundância e riqueza de anuros em fase larval nos locais estudados. Desta forma, a segregação apresentada pela anurofauna da Depressão Central e da Serra do Sudeste tende a estar relacionada com as diferenças nas varáveis ambientais e das poças que agem como reguladores da presença tanto de adultos quanto de larvas em cada uma das comunidades (Iop et al., 2011).

Estes mecanismos reguladores podem se apresentar nos anuros (adultos e larvas) na forma de distúrbios de comportamento e/ou disfunções fisiológicas (MCDiARmid \& Altig, 1999) como hipóxia, em decorrência de variações na taxa de $\mathrm{O}_{2}$ dissolvido na água (COSTA, 1967; WASSERSUG \& FEDER, 1983), modificações na colonização dos corpos d'água, pelo aumento/ diminuição do número de microambientes como sítios reprodutivos ou de abrigo (Kiesecker, 1996; Conte \& Machado, 2005; Moresco et al., 2009; AQuino et al., 2010) até situações mais danosas ao próprio desenvolvimento embrionário, como a variação do pH e da concentração de íons na água (PIERCE, 1985; KIESECKER, 1996; ver revisão completa em Di-BERNARDo et al., 2002).

Fatores relacionados à sazonalidade climática, como temperatura (tanto baixa quanto alta), também são conhecidos por agirem de forma reguladora sobre a presença ou a ausência dos anuros como por exemplo Hypsiboas pulchellus, que tem atividade pronunciada nos meses mais frios (López et al., 2011; ANTONIAZZI et al., 2013), podendo também afetar no regime hídrico beneficiando espécies como Rhinella schneideri nos meses mais secos (ODA et al., 2009) e Physalaemus gracilis e Scinax uruguayus nas intermitências chuvosas (Achaval \& Olmos, 2007; KwET et al., 2010). Estes fatores também são responsáveis por variações no hidroperíodo das poças que trazem, através da sua variação, algumas espécies que dependem destes momentos para reprodução (e.g. Leptodactylus fuscus) e por consequência modificações no uso dos estratos vegetacionais, tanto das margens quanto da cobertura do espelho d'água, beneficiando espécies como Physalaemus biligonigerus e Scinax squalirostris (ACHAVAL \& Olmos, 2007; ZARANCHO et al., 2004; KWET et al., 2010).

Como sugerido por Gambale et al. (2014) e Conte \& RosSA-FERES (2006), esta sazonalidade somada a constantes ações antrópicas (tipicamente encontradas nos ambientes abertos do bioma Pampa) acabam sendo mecanismos que indiretamente geram alterações tanto nas pressões predativas e competitivas quanto na própria disponibilidade de locais para ovoposição e desenvolvimento das larvas (FEDER, 1982; Woodward, 1983; Skelly, 1996; McDiarmid \& Altig, 1999; FAHD et al., 2000; Townsend, 2002), tornando os ambientes ainda mais susceptíveis à colonização pelas espécies generalistas como as encontradas.

Assim, os fatores como a alta permeabilidade da pele, a ectotermia e a dependência da água, em pelo menos um estágio de desenvolvimento, tornam o grupo dos anfíbios anuros especialmente sensível às variações dos parâmetros ambientais e da água, de forma que estas podem ser refletidas como variações na estrutura, na riqueza e nos padrões de distribuição das comunidades (DuELLMAN \& Trueb, 1994; McDiarmid \& Altig, 1999; Urbina-Cardona et al., 2006).

Em uma visão mais abrangente, as formações em mosaico (campo/floresta), nas duas regiões, algumas destas formações naturais da paisagem, outras de origem antrópica, podem apresentar uma estrutura de comunidade alterada, e um dos principais motivos para isto é modificação dos níveis de distúrbios no ambiente (PianKa, 1994). Assim como ocorre nas áreas de Cerrado, as comunidades de anuros do Pampa parecem ser beneficiadas pela heterogeneidade horizontal da paisagem, proporcionada pela variação no número de ambientes disponíveis, demonstrando então a importância da manutenção destas fisionomias campestres e da criação e implementação de unidades de conservação nestes locais já fragilizados pela ação antrópica.

Agradecimentos. Agradecemos à Coordenação de Aperfeiçoamento de Pessoal de Nível Superior (CAPES) pela bolsa de Mestrado concedida; aos agricultores e proprietários que permitiram o acesso às suas propriedades; ao ICMBio e ao SISBIO (29508/3) pelas licenças de coleta e transporte de material. Também agradecemos a Marcelo Rocha, Samanta Iop, Vinícius Caldart e Bruno Madalozzo pela ajuda no em laboratório e nas campanhas de amostragem. TGS agradece ao $\mathrm{CNPq}$ pela bolsa de pesquisa.

\section{REFERÊNCIAS BIBLIOGRÁFICAS}

Achaval, F. \& Olmos, A. 2007. Anfibios y Reptiles del Uruguay. Montevidéo, Biophoto. 160p.

Antoniazzi, C. E.; López, J. A.; Duré, M. \& Falico, D. A. 2013. Alimentación de dos especies de anfibios (Anura: Hylidae) en la estación de bajas temperaturas y su relación con la acumulación de energía en Santa Fe, Argentina. Revista de Biología Tropical 61(2):875-886.

Aquino, L.; Kwet, A.; Silvano, D.; Lavilla, E. \& Baldo, D. 2010. Scinax squalirostris. The IUCN Red List of Threatened Species. Version 2014.3. Disponível em <www.iucnredlist.org>. Acesso em 14 dezembro 2014.

Bastazini, C. V.; Munduruca, J. F.; Roch, P. L. B. \& Napoli, M. F. 2007. Which environmental variables better explain changes in anuran community composition? A case study in the restinga of Mata de São João, Bahia, Brazil. Herpetologica 63(4):459-471.

Behling, H.; Jeske-Pieruschka, V.; Schüler, L. \& Pillar, V. 2009. Dinâmica dos campos no sul do Brasil durante o Quaternário Tardio. In: Pillar, V; Müller, S. C.; Souza Castilhos, Z. M. \& Jacques, A. V. A. eds. Campos Sulinos - Conservação e Uso Sustentável da Biodiversidade. Brasília, Ministério do Meio Ambiente, p. 13-25.

Bencke, G. A.; Jardim, M. M. A.; Borges-Martins, M. \& Zank, C. 2009. Composição e padrões de distribuição da fauna de tetrápodes recentes do Rio Grande do Sul, Brasil. In: Ribeiro, A. M.; Bauermann, S. G.; SCHERER, C. S. eds. Quaternário do Rio Grande do Sul, Integrando Conhecimentos. Porto Alegre, Monografias da Sociedade Brasileira de Paleontologia, p.123-142. 
BoldRINI, I. I. 1997. Campos do Rio Grande do Sul: caracterização fisionômica e problemática ocupacional. Boletim do Instituto de Biociências/UFRGS 56:1-39.

Boldrini, I. I.; Ferreira, P. M. A.; Andrade, B. O.; Schneider, A. A.; Setubal, R. B.; Trevisan, R. \& Freitas, E. M. 2010. Bioma Pampa: Diversidade florística e fisionômica. Porto Alegre, Pallotti. 64p.

Both, C.; Melo, A. S.; Cenchin, S. Z. \& Hartz, S. M. 2011. Tadpole co-occurrence in ponds: When do guilds and time matter? Acta Oecologica 37:140-145.

Both, C.; Solé, M.; Santos, T. G. \& Cechin, S. Z. 2009. The role of spatial and temporal descriptors for neotropical tadpole communities in southern Brazil. Hydrobiologia 624:125-38.

Camargo, A. N.; Canavero, D. E.; Andrés, R. I. \& Maneyro, R. 2005. Seasonal activity and the body size-fecundity relationship in a population of Physalaemus gracilis (Boulenger, 1883) (Anura, Leptodactylidae) from Uruguay. Annales Zoological Fennici 42:513-521

CLARK, K. R. \& WARWICK, R. M. 2001a. Change in marine communities: an approach to statistical analysis and interpretation. 2ed. Plymouth, Primer-E. 172p.

2001b. PRIMER v5: User manual. Plymouth, Primer-E.

Clarke, K. \& Ainsworth, M. 1993. A method of linking multivariate community structure to environmental variables. Marine EcologyProgress Series 92:205-219.

Colombo, P.; Kindel, A.; Vinciprova, G. \& Krause, L. 2008. Composição e ameaças à conservação dos anfíbios anuros do Parque Estadual de Itapeva; município de Torres; Rio Grande do Sul; Brasil. Biota Neotropica 8:229-240.

Conte, C. E. \& Machado, A. R. 2005. Riqueza de espécies e distribuição espacial e temporal em comunidades de anuro (Amphibia; Anura) em uma localidade de Tijucas do Sul, Paraná, Brasil. Revista Brasileira de Zoologia 22(4):940-948.

Conte, E. C. \& Rossa-Feres, D. 2006. Diversidade e ocorrência temporal da anurofauna (Amphibia, Anura) em São José dos Pinhais, Paraná, Brasil. Revista Brasileira de Zoologia 23(1):162-175.

Cordeiro, J. L. P. \& Hasenack, H. 2009. Cobertura vegetal atual do Rio Grande do Sul. In: Pillar, V. P; Müller, S. C.; Castilhos, Z. M. $S$ \& JACQues, A. V. A. eds. Campos Sulinos: conservação e uso sustentável. Brasília, Ministério do Meio Ambiente, p. 285-299.

Costa, H. H. 1967. Avoidance of anoxic water by tadpoles of Rana temporaria. Hydrobiologia 30(3-4):374-384.

Di-Bernardo, M.; Kwet, A.; Teixeira, E. C. \& Pires, M. J. R. 2002. Efeitos da contaminação de águas superficiais associadas a atividades de extração e processamento de carvão sobre anfíbios. In: TEIXEIRA, E. C.; Pires, M. J. R. eds. Meio ambiente e carvão. Impactos da exploração e utilização. Porto Alegre, PADCT/GTM/FINEP/ CAPES/PUCRS/UFSC/FEPAM, p. 413-422.

Duellman, W. E. \& TRUeB, L. 1994. Biology of amphibians. Baltimore, The John Hopkins University Press. 670p.

DufrÊNe, M. \& LegGendRE, P. 1997. Species assemblages and indicator species: the need for a flexible asymmetrical approach. Ecological Monographs 67:345-366.

Fahd, K.; Serano, L. \& ToJa, J. 2000. Crustacean and Rotifer Composition of Temporary Ponds in the Doñana National Park (SW Spain) During Floods. Hydrobiologia 436:41-49.

Feder, M. E. 1982. Effect of developmental stage and body size on oxygen consumption on anuran larvae: A reappraisal. Journal of Experimental Zoology 220:33-42.

Gambale, P. G.; Woitovicz-Cardoso, M.; Vieira, R. R.; Batista, G. V.; Ramos, J. \& Bastos, R. P. 2014. Composição e riqueza de anfíbios anuros em remanescentes de Cerrado do Brasil Central. Iheringia, Série Zoologia 104(1):50-58.

Garcia, P. C.; Faivovich, J. N. \& Haddad, C. F. B. 2007. Redescription of Hypsiboas semiguttatus with the description of a new species of the Hypsiboas pulchellus group. Copeia 4:933-949.

Gautreau, P. \& VéLez, E. 2011. Strategies of environmental knowledge production facing land use changes: Insights from the Silvicultural Zoning Plan conflict in the Brazilian state of Rio Grande do Sul. Cybergeo: European Journal of Geography 577.

Gotelli, N. J. \& Colwell, R. K. 2001. Quantifying biodiversity: procedures and pitfalls in the measurement and comparison of species richness. Ecology Letters 4:379-391.
GotTSBerger, B. \& GruBer, E. 2004. Temporal partitioning of reproductive activity in a neotropical anuran community. Journal of Tropical Ecology 20:271-280.

Guadagnin, D. L.; Zalba, S.; Górriz, B.; Fonseca, C.; NebBia, A. J.; Cuevas, Y.A.; Emer, C.; Germain, P.; Wendland, E. M. D. R; Perello, L. F. C.; Bastos, M. C. S.; Sanhueza, C. C.; Masciadri-Bálsamo, S. \& Villalobos, A. E. 2009. Árvores e arbustos exóticos invasores no Pampa: questões ecológicas; culturais e sócio-econômicas de um desafio crescente. In: Pillar V. P.; Müller, S. C.; Castilhos, Z. M. S. \& JACQUES, A. V. A. eds. Campos Sulinos: conservação e uso sustentável da biodiversidade. Brasília, MMA. 403p.

Hasenack, H.; Cordeiro, J. L. P. \& Costa, B. S. C. 2007. Cobertura vegetal atual do Rio Grande do Sul. In: DALL'AGNOL, M.; NABINGER, C.; Sant'Anna, D. M. \& Santos, R. J. eds. II Simpósio de Forrageiras e Produção Animal. Porto Alegre, Depto. Forrageiras e Agrometeorologia/UFRGS, p. 15-21.

Hasenack, H.; Weber, E.; Boldrini, I. I. \& Trevisan, R. 2010. Mapa de sistemas ecológicos da ecorregião das savanas uruguaias em escala 1: 500.000 ou superior e relatório técnico descrevendo insumos utilizados e metodologia de elaboração do mapa de sistemas ecológicos. Porto Alegre, UFRGS, Centro de Ecologia, The Nature Conservancy.

Hawkins, M. A.; Sites JR., J. W. \& Noonan, B. P. 2007. Dendropsophus minutus (Anura: Hylidae) of the Guiana Shield: using DNA barcodes to assess identity and diversity. Zootaxa 1540:61-67.

Hero, J. M.; Gascon, C. \& Magnusson, W. E. 1998. Direct and indirect effects of predation on tadpole community structure in the Amazon rainforest. Austral Ecology 23(5):474-482.

Iop, S.; Caldart, V. M.; Santos, T. G. \& Cechin, S. Z. 2011. Anurans of Turvo State Park: testing the validity of Seasonal Forest as a new Biome in Brazil. Journal of Natural History 45:2443-2461.

IUCN. 2012. IUCN Red List of Threatened Species. Version 2012.1. Disponível em $<$ www.iucnredlist.org $>$.

KIESECKER, J. M. 1996. pH induced growth reduction and its effects on predator-prey interactions between Ambystoma tigrinum and Pseudacris triseriata. Ecology Applied 6:1325-1331.

Krapovickas, S. \& Di Giacomo, A. S. 1998. Conservation of pampas and grasslands in Argentina. Parks 8:47-53.

Kwet, A. \& Di-Bernardo, M. 1998. Elachistocleis erythrogaster; a new microhylid species from Rio Grande do Sul; Brazil. Studies on Neotropical Fauna and Environment 33:7-18.

Kwet, A.; Lignau, R. \& Di-Bernardo, M. 2010. Pró-Mata: Anfíbios da Serra Gaúcha, sul do Brasil - Amphibien der Serra Gaúcha, Südbrasilien - Amphibians of the Serra Gaúcha, South of Brazil. 2ed. Tübingen, Brasilien Zentrum der Universität Tübingen. 148p.

López, J. A.; Scarabotti, P. A. \& Ghirardi, R. 2011. Seasonal patterns of abundance and recruitment in an amphibian assemblage from the Paraná River floodplain. Interciência 36:538-544.

Machado, I. F. \& MaLTchiK, L. 2009. Can management practices in rice fields contribute to amphibian conservation in southern Brazilian wetlands? Aquatic Conservation: Marine and Freshwater Ecosystems 20:39-46.

Machado, I. F.; Moreira, L. F. \& Maltchik, L. 2012. Effects of pine invasion on anurans assemblage in southern Brazil coastal ponds. Amphibia-Reptilia 33:227-237.

Maltchik, L.; Peixoto, C.; Stenert, C.; Moreira, L. \& Machado, I. 2008. Dynamics of the terrestrial amphibian assemblage in a flooded riparian forest fragment in a Neotropical region in the south of Brazil. Brazilian Journal of Biology 68:763-769.

Maluf, J. R. T. 1999. Nova classificação climática do Estado do Rio Grande do Sul. Passo Fundo, Embrapa Trigo. 9p.

Maneyro, R. \& Langone, J. A. 2001. Categorización de los anfibios de Uruguay. Cuadernos de Herpetología 15(2):107-118.

McDiarmid, R. W. \& Altig, R. 1999. Tadpoles: The Biology of Anuran Larvae. Chicago, University of Chicago Press. 444p.

Moreira, L.; Machado, I.; Lace, A. \& Maltchik, L. 2008. Anuran amphibians dynamics in an intermittent pond in Southern Brazil. Acta Limnologica Brasiliensia 20:205-212.

Moreira, L. F. B. \& MaltChiK, L. 2012. Assessing patterns of nestedness and co-occurrence in coastal pond anuran assemblages. AmphibiaReptilia 33:261-271. 
Moresco, R. M; Margarido, V. P; Nazario, P. D; Schmit, R. A \& Treco, F. R. 2009. Amphibia; Anura; Hylidae; Scinax granulatus: Distribution extension. Check List 5:086-088.

Nogueira, C.; Colli, G. R. \& Martins, M. 2009. Local richness and distribution of the lizard fauna in natural habitat mosaics of the Brazilian Cerrado. Austral Ecology 34(1):83-96.

Oda, F. H.; Bastos, R. P. \& SÁ Lima, M. A. D. C. 2009. Taxocenose de anfíbios anuros no Cerrado do Alto Tocantins, Niquelândia, estado de Goiás: diversidade, distribuição local e sazonalidade. Biota Neotropica 9(4):219-232.

Oliveira, M. C. L. M.; Santos, M. B; Loebmann, D.; Hartman, A. \& Tozetri, A. M. 2013. Diversity and associations between coastal habitats and anurans in southernmost Brazil.Anais da Academia Brasileira de Ciências 58(2):575-583.

Overbeck, G. E.; Müller, S. C.; Fidelis, A.; Pfadenhauer, J.; Pillar, V. D.; Blanco, C. C.; Boldrini, I. I; Both, R. \& Forneck, E. D. 2007. Brazil's neglected biome: The South Brazilian Campos. Perspectives in Plant Ecology; Evolution and Systematics 9:101-116.

PiAnKa, E. R. 1994. Evolutionary Ecology. New York, Harper Collins College Publisher. 486p.

Pierce, B. A. 1985. Acid tolerance in amphibians. BioScience 35(4):239243.

Porto, M. 2002. Os campos sulinos: sustentabilidade e manejo. Ciência \& Ambiente 24:119-138.

Quintela, F. M.; Neves, L. F. M.; Medvedovisky, I. G.; Santos, M. B.; Oliveira, M. C. L. M. \& Figueiredo, M. R. C. 2009. Relação dos anfíbios da Ilha dos Marinheiros; estuário da Lagoa dos Patos; Rio Grande do Sul; Brasil. Revista Brasileira de Biociências 7(2):231233.

R Development Core Team. 2012. R: A language and environment for statistical computing. Vienna, R Foundation for Statistical Computing. Disponível em: <http://www.R-project.org/>.

Rambo, B. 1956. A fisionomia do Rio Grande do Sul. Porto Alegre, Livraria Selbach. 360p.

Santos, T. G; Kopp, K.; Spies, M. R.; Trevisan, R. \& Cechin, S. Z. 2008. Distribuição temporal e espacial de anuros em área de Pampa, Santa Maria, RS. Iheringia, Série Zoologia 98(2):244-253.

Santos, T. G; Rossa-Feres, D. C. \& Casatti, L. 2007. Diversidade e distribuição espaço-temporal de anuros em região com pronunciada estação seca no sudeste do Brasil. Iheringia, Série Zoologia 97(1):3749.

Scott Jr, N. J. \& Woodward, B. D. 1994. Surveys at breeding sites. In: Heyer, W. R.; Connelly, M. A; McDiarmid, R. W.; Hayek, L. A. C. \& Foster, M. S. orgs. Measuring and Monitoring Biological Diversity - Standard Methods for Amphibians. Washington, Smithsonian Institution Press, p. 84-92.

Silva R. A.; Martins, I. A. \& Rossa-Feres, D. C. 2011. Environmental heterogeneity: Anuran diversity in homogeneous environments. Zoologia 28(5):610-618.
Skelly, D. K. 1996. Pond drying; predators; and the distribution of Pseudacris tadpoles. Copeia 1996:599-605.

TownsEnd, S. A. 2002. Seasonal evaporative concentration of an extremely turbid water body in the semiarid tropics of Australia. Lakes \& Reservoir: Research and Management 7:103-107.

Trindade, A. O.; Oliveira, S. V. \& Cappellari, L. H. 2010. Anfíbios anuros de uma área da Serra do Sudeste, Rio Grande do Sul (Caçapava do Sul). Biodiversidade Pampeana 8(1):14-24.

Urbina-Cardona, J. N.; Olivares-Pérez, M. \& Reynoso, V. H. 2006. Herpetofauna diversity and microenvironment correlates across a pasture-edge-interior ecotone in tropical rainforest fragments in the Los Tuxtlas Biosphere Reserve of Veracruz; Mexico. Biological Conservation 132:61-75.

Valdujo, P. H.; Camacho, A.; Recoder, R. S.; Teixeira, M.; Ghellere, J. M. B; Mott, T.; Nunes, P. M. S.; Nogueira, C. \& Rodrigues, M. T. U. 2011. Anfíbios da Estação Ecológica Serra Geral do Tocantins, região do Jalapão, estados do Tocantins e Bahia. Biota Neotropica 11(1):251-262.

VAsconcelos, T. S. \& Rossa-Feres, D. C. 2005. Diversidade, distribuição espacial e temporal de anfíbios anuros (Amphibia, Anura) na região noroeste do Estado de São Paulo, Brasil. Biota Neotropica 5:1-14.

2008. Habitat heterogeneity and use of physical and acoustic space in anuran communities in southeastern Brazil. Phyllomedusa 7(2):127-142

Vasconcelos, T. S.; Santos T. G.; Rossa-Feres, D. C. \& Haddad, C. F. B. 2009. Influence of the environmental heterogeneity of breeding ponds on anuran assemblages from southeastern Brazil. Canadian Journal of Zoology 87:699-707.

2010. Similarity of ground-dwelling anuran (Amphibia) composition among different vegetation physiognomies in a Mesophytic Semideciduous Forest from southeastern Brazil. NorthWestern Journal of Zoology 6:275-285.

Vieira, E. F \& Rangel, S. R. S. 1984. Rio Grande do Sul: geografia física e vegetação. Porto Alegre, Sagra. 256p.

Wassersug, R. J. \& Feder, M. E. 1983. The effects of aquatic oxygen concentration; body size and respiratory behaviour on the stamina of obligate aquatic (Bufo americanus) and facultative air-breathing (Xenopus laevis and Rana berlandieri) anuran larvae. Journal of Experimental Biology 105:173-190.

WoODWARD, B. D. 1983. Predator-prey interactions and breeding-pond use of temporary species in a desert anuran community. Ecology 64:1549-1555.

Xavier, A. L \& Napoli, M. F. 2011. Contribution of environmental variables to anuran community structure in the Caatinga Domain of Brazil. Phyllomedusa 10:45-64.

Zarancho, V. H.; Céspedez, J. A. \& Álvarez, B. B. 2004. Caracterización de las puestas de especies del género Physalaemus (Anura: Leptodactylidae) en Argentina. Boletín de la Asociación Herpetológica Española 15:100-104. 